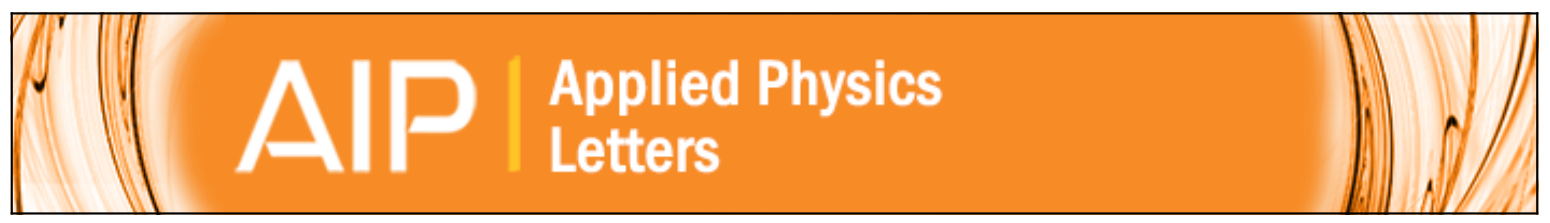

\title{
Basins of attraction of tapping mode atomic force microscopy with capillary force interactions
}

Nastaran Hashemi and Reza Montazami

Citation: Applied Physics Letters 94, 251902 (2009); doi: 10.1063/1.3148672

View online: http://dx.doi.org/10.1063/1.3148672

View Table of Contents: http://scitation.aip.org/content/aip/journal/apl/94/25?ver=pdfcov

Published by the AIP Publishing

\section{Articles you may be interested in}

The effect of set point ratio and surface Young's modulus on maximum tapping forces in fluid tapping mode atomic force microscopy

J. Appl. Phys. 107, 044508 (2010); 10.1063/1.3309330

The dissipated power in atomic force microscopy due to interactions with a capillary fluid layer

J. Appl. Phys. 104, 063518 (2008); 10.1063/1.2980057

The nonlinear dynamics of tapping mode atomic force microscopy with capillary force interactions J. Appl. Phys. 103, 093512 (2008); 10.1063/1.2913054

Special cantilever geometry for the access of higher oscillation modes in atomic force microscopy Appl. Phys. Lett. 89, 033106 (2006); 10.1063/1.2226993

Spectroscopy of the anharmonic cantilever oscillations in tapping-mode atomic-force microscopy Appl. Phys. Lett. 77, 3293 (2000); 10.1063/1.1325404

\section{AlP Re-register for Table of Content Alerts}




\title{
Basins of attraction of tapping mode atomic force microscopy with capillary force interactions
}

\author{
Nastaran Hashemi ${ }^{1, a)}$ and Reza Montazami ${ }^{2}$ \\ ${ }^{1}$ Department of Mechanical Engineering, Virginia Polytechnic Institute and State University, \\ Blacksburg, Virginia 24061, USA \\ ${ }^{2}$ Department of Materials Science and Engineering, Virginia Polytechnic Institute and State University, \\ Blacksburg, Virginia 24061, USA
}

(Received 24 March 2009; accepted 14 May 2009; published online 22 June 2009)

\begin{abstract}
We perform a large number of simulations over a wide range of system parameters to approximate the basins of attraction of steady oscillating solutions. We find that the basins of attraction vary as a function of system parameters and initial conditions. For large equilibrium separations, the basin of attraction is dominated by the low-amplitude solution. The location of the fixed point is shifted toward the higher values of instantaneous displacement and velocity for larger equilibrium separations. We show that the basin of attraction in the neighborhood of the fixed point is dominated by low-amplitude solutions as relative humidity is increased. (C) 2009 American Institute of Physics. [DOI: $10.1063 / 1.3148672$ ]
\end{abstract}

The increased application of micro/nanoelectromechanical systems has lead to the importance of understanding nonlinear dynamics at the micro/nanoscale. One potential benefit is that the sensitivity and precision of some measurements can be improved while the system is driven in nonlinear region. ${ }^{1-3}$ Atomic force microscopy (AFM) has revolutionized surface science with its ability to generate topographical mappings with atomic scale resolution. ${ }^{4,5}$ In tapping mode the interaction forces between the AFM tip and the sample are discontinuous, nonlinear, and hysteretic. ${ }^{6-8}$ For example, for a given set of parameters there often exist multiple solutions to equation of motion. ${ }^{6}$ As a result, the oscillating AFM cantilever can switch between solutions and it is often the case that one solution will have a larger impact velocity, which can be detrimental to the sample. ${ }^{5}$ The bistable behavior is due to the attractive and repulsive tip sample interactions. Zitzler et al. ${ }^{6}$ explored the role of capillary forces resulting from humidity.

The objective of this research is to quantify the basins of attraction of AFM cantilevers in tapping-mode operation with the inclusion of capillary force interactions. Similar studies have been performed in the absence of the capillary force and have uncovered the coexistence of multiple steady state solutions with complex basins of attraction. ${ }^{5,9}$ However, it is not clear beforehand if these findings remain with the inclusion of the capillary fluid layer that is present in experiment. We determined all the possible steady state solutions and approximated the basins of attraction of these attractors in order to build an understanding of how these attractors change as system parameters are varied. Of particular interest were the variation of the equilibrium separation of the cantilever from the surface during surface scanning and the variation of water film thickness on the sample and cantilever tip surfaces. The deterministic character of the equation of motion requires that when a steady state attractor is found, the AFM cantilever oscillates there, independent of the extent of its basin of attraction. Small perturbations can influence the dynamics of a system significantly. For numerical

${ }^{a)}$ Electronic mail: nastaran@vt.edu. simulations, small perturbations exist due to the particulars of the numerical scheme as well as round-off error. Garcia and San Paulo ${ }^{10}$ explored the basin of attraction in the absence of the capillary fluid layer and uncovered a complex pattern of low and high amplitude steady state periodic solutions as the equilibrium distance of the cantilever is varied. We have performed forward-time simulations using event handling techniques tailored for use with discontinuous systems to quantify the basin of attraction..$^{8,11,12}$ The corresponding equation of motion is a nonlinear, second order ordinary differential given by, ${ }^{6}$

$$
m \ddot{q}+c \dot{q}+k q=F_{d} \cos \omega_{d} t+F_{\text {int }} .
$$

$F_{\text {int }}$ is the tip-sample interaction force, which depends on the excursion $q$ of the tip relative to a reference level and its past motion. We have found low and high amplitude periodic solutions, and irregular or high-periodic motion. Figure 1 shows the minimum distance $d$ between the mass and the surface for the final 200 oscillations of the cantilever. We

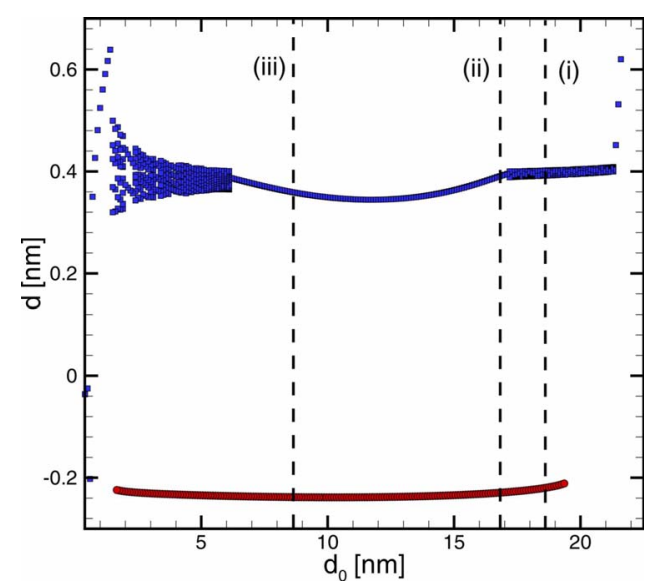

FIG. 1. (Color online) The variation of the closest separation distance $d$ between the cantilever and the surface with equilibrium separation $d_{0}$. When plotted in this way the scatter in the results indicates the presence of windows of irregular or high-periodic dynamics. The vertical dashed lines indicate the three separations used in calculating projections of the basin of attraction. 


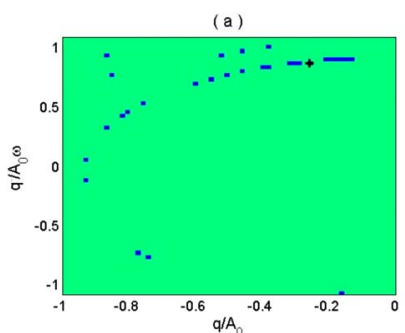

(b)

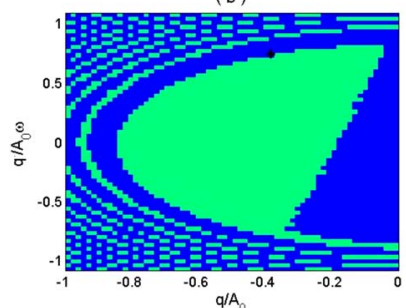

(c)

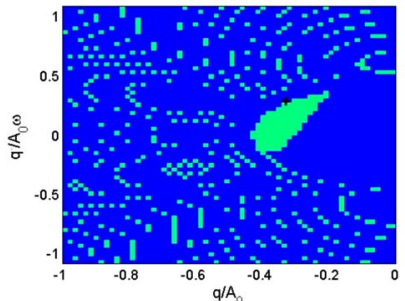

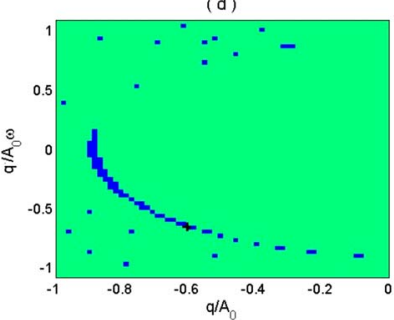

(e)

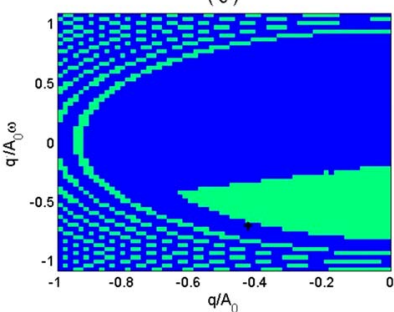

(f)

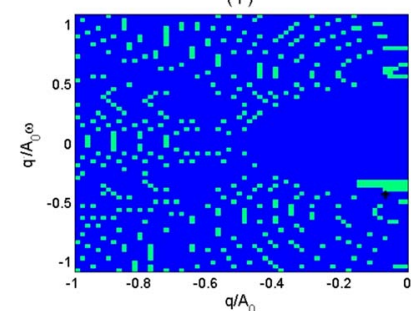

FIG. 2. (Color online) Two-dimensional projections of the basin of attraction of steady oscillating solutions for capillary layer thickness of $h$ $=0.2 \mathrm{~nm}$. Large separation $d_{0}=18.6 \mathrm{~nm}[(\mathrm{a})$ and (d)], intermediate separation $d_{0}=16.9 \mathrm{~nm}\left[(\mathrm{~b})\right.$ and (e)], and small separation $d_{0}=8.7 \mathrm{~nm}[(\mathrm{c})$ and (f)]. Left column shows $x(t=0)=(q, \dot{q}, 0)$ and right column shows $x(t=0)$ $=(q, \dot{q}, 4 \pi / 3)$. Blue rectangles represent initial conditions that yield highamplitude periodic solutions and green rectangles represent initial conditions that yield low-amplitude periodic solutions. Theoretical fixed points are indicated by black cross.

would like to highlight that our numerical simulations did not jump from one stable solution branch to another as we changed equilibrium separation, $d_{0}$. This is a result of our careful use of event handling techniques, which incur very little numerical perturbation to the solution.

We have computed two-dimensional projections of the basin of attraction for the three different equilibrium cantilever separations. For each separation we have performed simulations over a range of initial conditions in initial displacement $q(0)$ and velocity $\dot{q}(0)$ for initial phases of $\phi=0$ and $\phi=4 \pi / 3$. Considering the three-dimensional continuous state vector, these results correspond to two separate planes in the three-dimensional space. The basins of attraction for capillary layer thickness of $h=0.2 \mathrm{~nm}$ are shown in Fig. 2. The grid spacing in initial displacement is $\Delta q_{0}=0.323 \mathrm{~nm}$, the grid spacing in initial velocity is $\Delta \dot{q}_{0}=1.23 \mathrm{~mm} / \mathrm{s}$, and the bottom left of each colored rectangle represents the value of the initial conditions used in the simulation. Green indicates the low-amplitude periodic solution and blue represents the high-amplitude periodic solution. Theoretical fixed points are indicated by black cross. Because the computation of the basins of attraction are performed only for the negative values of $q$ and the fixed points of the low-amplitude solution are located in the positive values of $q$, only the fixed point for the high-amplitude solution are shown in Fig. 2. Further investigations of the location of the fixed points show that the fixed points are located near the low and high-amplitude solution boundary. Figures 2(a)-2(c) represent our numerical
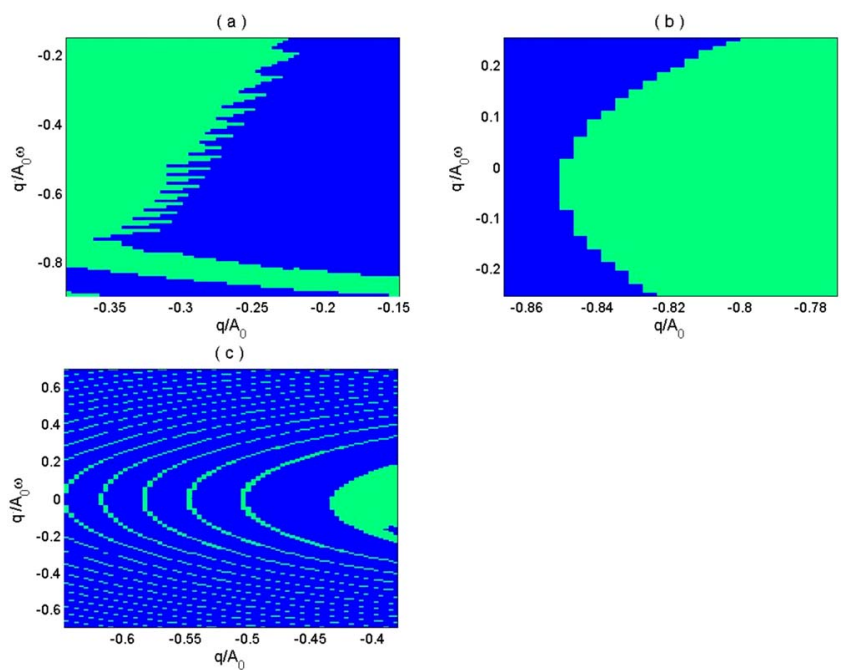

FIG. 3. (Color online) [(a) and (b)] Simulations with higher resolution in the grid of initial conditions for two regions shown in Fig. 2(b). (c) Simulations with higher resolution in the grid of initial conditions to explore in more detail a portion of Fig. 2(c). The branches of the low-amplitude solution are connected and the dynamics is quite complicated.

results for initial phase of $\phi=0$. Figure 2(a) illustrates that for large separations the low-amplitude periodic solution is very stable and dominates basin of attraction projection. For the separation of $d_{0}=18.6 \mathrm{~nm}$, the low-amplitude solution is found to be high periodic as well [see the dashed vertical line labeled (i) in Fig. 1]. Figure 2(b) illustrates that for intermediate separations there is a large portion of the phase space projection dominated by the low-amplitude periodic solution. However, outside this region there is a complicated pattern of initial conditions leading to the high and low-amplitude periodic solutions indicating a significant decrease in stability. Previous studies performed by Garcia and San Paulo ${ }^{10}$ present a simpler dynamics outside the region dominated by the low-amplitude solution. Including the capillary force interactions, as we do here, results in more complicated dynamics. For example, for the same region of phase space, there exist several circular regions of low-amplitude solutions separated by several regions of high-amplitude solutions compared to only one circular branch found by Garcia and San Paulo. ${ }^{10}$ Figures 3(a) and 3(b) represent higher resolution simulations for two separate regions of the Fig. 2(b). The grid spacing in displacement and initial velocity are $\Delta q_{0}=0.082 \mathrm{~nm}$ and $\Delta \dot{q}_{0}=0.312 \mathrm{~mm} / \mathrm{s}$, respectively. Using this resolution we find that the basin boundaries are nonsmooth. Fractal basins are found in dissipative systems and contain open sets separated by nonsmooth boundaries. ${ }^{13}$ Figure 3(b) also reveals that the basin of attraction is nonsymmetric. These investigations provide a clearer insight about the dynamics of the AFM. Figure 2(c) yields that for small separations the stability of the low-amplitude solution is lost. The basin of attraction is now dominated by initial conditions leading to the high-amplitude solution. However, the initial conditions leading to the low-amplitude solution are quite spread out and do not appear connected at this resolution. Again, we have studied a portion of the phase space with higher resolution. As shown in Fig. 3(c), the branches of the low-amplitude solution are found to be connected. The dynamics is quite complicated. ${ }^{10}$ Figures $2(\mathrm{~d})-2(\mathrm{f})$ represent our numerical results for initial phase of $\phi=4 \pi / 3$. Figure 2 (d) shows that the low-amplitude solution dominates basin 

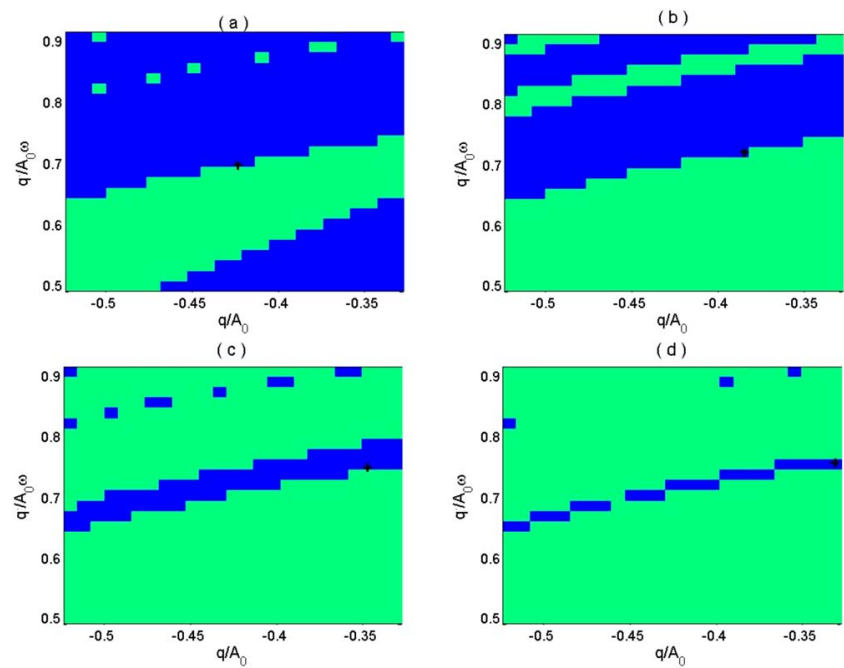

FIG. 4. (Color online) Basins of attraction in some neighborhood of the theoretical fixed points for different values of the capillary layer thickness $h=0.1$ (a), 0.2 (b), 0.3 (c), and $0.4 \mathrm{~nm}$ (d).

of attraction projection the same as Fig. 2(a). However, Fig. 2(e) illustrates that the large portion of the phase space dominated by the low-amplitude periodic solution in Fig. 2(b) is replaced by the high-amplitude periodic solution while outside this region shows the same pattern. Figure $2(\mathrm{f})$ yields that for this initial phase the central portion of the phase space is dominated by the high-amplitude solution as opposed to the central portion of the Fig. 2(c).

It is important to understand that for the same separation $d_{0}$, the two plots for different initial phase $\phi$ are different. These results suggest that the basin boundary is not a cylinder parallel to $\phi$. Our results further confirm previous findings by Marth et al. ${ }^{9}$ The basins of attraction are in general different for different system parameters. In this letter, we have studied the basins of attraction by varying three different parameters $P=\left\{d_{0}, \phi, h\right\}$. To follow up our previous study on the effect of humidity on the dynamics of the tapping mode AFM, ${ }^{11}$ we have explored how the basins of attraction vary with the variation of humidity $h$. Figures 4(a)-4(d) represent the basins of attraction for different values of the capillary layer thickness $h=0.1$ (a), 0.2 (b), 0.3 (c), and $0.4 \mathrm{~nm}$ (d) in some neighborhood of the theoretical fixed points indicated by black cross. The equilibrium separation is $d_{0}$ $=16.9 \mathrm{~nm}$ for these simulations. As $h$ increases further the low-amplitude solutions dominate the basin of attraction in the same neighborhood around the fixed point and also the fixed point itself moves toward the higher values of the $q$ and $\dot{q}$. We have also shown the variation of the fixed point location for three values of $d_{0}$ in Fig. 5. As $d_{0}$ is increased from 16.9 to $18.6 \mathrm{~nm}$, the fixed points of the high amplitude solutions shift toward the higher values of the $q$ and $\dot{q}$. Since for larger equilibrium separation the low amplitude solution is dominant, it takes higher values of $q$ and $\dot{q}$ for the system to jump to the high amplitude solution. The same trends are found as $d_{0}$ is increased from 8.7 to $16.9 \mathrm{~nm}$ for the capillary layer thickness of $h=0.3$ and $0.4 \mathrm{~nm}$. However for lower $h$ fixed points shift toward higher values of $\dot{q}$ and lower $q$ values. Also, the fixed points of the high amplitude solutions shift toward the higher values of the $q$ and $\dot{q}$ as $h$ is increased.

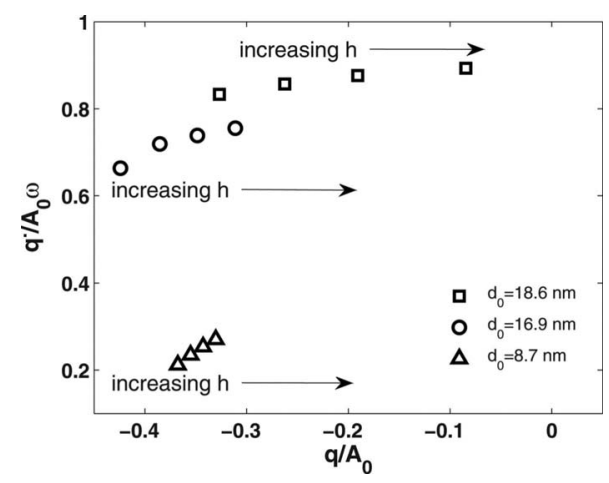

FIG. 5. The variation in the location of the fixed point as a function $d_{0}$ and $h$. The fixed points are computed for $d_{0}=18.6 \mathrm{~nm}$ (squares), $16.9 \mathrm{~nm}$ (circles), $8.7 \mathrm{~nm}$ (triangles) and for the capillary layer thickness of $h=0.1$, $0.2,0.3$, and $0.4 \mathrm{~nm}$.

While an AFM is scanning, the topographic features of an arbitrary sample can produce large variations in the rest tip surface separation before the steady oscillating solution is found. This is an intrinsic source of noise that is very significant in experiment. An analysis of this noise requires detailed information of the sample being explored.

In conclusion, using forward-time simulations that carefully treat the dynamics of this hybrid dynamical system we find the coexistence of three steady oscillating solutions. We have quantified two-dimensional projections of the basin of attraction for three different values of the equilibrium cantilever-surface separation, two different values of phase, and four different values of the capillary layer thickness. We have gained new insight into the effect of different system parameters on the basins of attraction of the tapping mode AFM. The basin of attraction is dominated by the lowamplitude solution at large equilibrium separations and by the high-amplitude periodic solution at small separations. Also, the low-amplitude solutions dominate the basin of attraction in the neighborhood of the fixed point as relative humidity increases. Furthermore, quantitative measurements of the basins of attraction should provide useful information for precision measurement applications in an ambient environment.

We thank Mark Paul and Harry Dankowicz for useful discussions. The early stages of this work were supported by NSF CMMI Grant No. 0510044 and 0619028, and NSF OISE Award No. 0714404.

${ }^{1}$ I. Kozinsky, H. W. Ch. Postma, O. Kogan, A. Husain, and M. L. Roukes, Phys. Rev. Lett. 99, 207201 (2007).

${ }^{2}$ E. Buks and B. Yurke, Phys. Rev. E 74, 046619 (2006)

${ }^{3}$ J. S. Aldridge and A. N. Cleland, Phys. Rev. Lett. 94, 156403 (2005).

${ }^{4}$ G. Binnig, C. F. Quate, and C. Gerber, Phys. Rev. Lett. 56, 930 (1986).

${ }^{5}$ R. Garcia and R. Perez, Surf. Sci. Rep. 47, 197 (2002).

${ }^{6}$ L. Zitzler, S. Herminghaus, and F. Mugele, Phys. Rev. B 66, 155436 (2002).

${ }^{7}$ H. Dankowicz, Philos. Trans. R. Soc. London, Ser. A 364, 3505 (2006).

${ }^{8}$ N. Hashemi, M. R. Paul, and H. Dankowicz, J. Appl. Phys. 103, 093512 (2008).

${ }^{9}$ M. Marth, D. Maier, J. Honerkamp, R. Brandsch, and G. Bar, J. Appl. Phys. 85, 7030 (1999).

${ }^{10}$ R. Garcia and A. San Paulo, Phys. Rev. B 61, R13381 (2000).

${ }^{11}$ N. Hashemi, M. R. Paul, H. Dankowicz, M. Lee, and W. Jhe, J. Appl. Phys. 104, 063518 (2008).

${ }^{12}$ N. Hashemi, Ph.D. Dissertation, Virginia Tech, 2008.

${ }^{13}$ J. Aguirre and M. A. F. Sanjuan, Phys. Rev. E 67, 056201 (2003). 